\title{
ANÁLISIS COMPARATIVO DE LAS TECNOLOGÍAS VCL Y FSO COMO GUÍA PARA LA TOMA DE DECISIONES DE INGENIE- ROS QUE PLANIFICAN REDES DE ACCESO
}

\author{
Camilo Andrés Hernández Torres \\ Programa de ingeniería de telecomunicaciones. Miembro \\ del semillero de investigación Fotón. Correo electrónico: \\ camilo-hernandez@upc.edu.co.
}

\section{Resumen}

Las comunicaciones móviles, durante los últimos años, han tenido un crecimiento significativo en su ancho de banda con respecto a la cantidad en el número de usuarios. La consecuencia inmediata es la implementación de otras tecnologías inalámbricas, las cuales soportan servicios integrados a las redes móviles y que de igual manera necesitan grandes anchos de banda para cubrir con la demanda tanto de usuarios conectados a internet como de las aplicaciones y servicios que a diario se desarrollan.

Surge entonces la posibilidad de usar otras tecnologías que utilizan luz visible para establecer la comunicación evitando algunos de los problemas que presentan las tecnologías cuyas portadoras están en la gama de las microondas y que se encuentran dentro de los sistemas de comunicaciones ópticas. Las tecnologías VLC (Visible Light Communications) y FSO (Free Space Optical) se usan para la transmisión de datos de alta velocidad a frecuencias portadoras entre 400 y $800 \mathrm{THZ}$.

\section{Introducción}

Uno de los retos más grandes que tienen los ingenieros de telecomunicaciones cuando son contratados como consultores o asesores en un nuevo proyecto tecnológico, es decidir la escogencia de la tecnología apropiada para un ámbito o servicio definido, dada la innumerable oferta que actualmente se tiene. En las tecnologías y medios usados en telecomunicaciones para redes de acceso o aquellas que prestan los servicios desde los nodos de distribución hasta el cliente, hay sobre oferta y es difícil tomar una decisión para indicarle a un cliente cuál es la mejor, a menos que se tengan muy claros los objetivos en cuanto a prestación de servicios, plataformas de gestión, facilidades en la operación y mantenimiento, integración, expansión, economía y obsolescencia, entre otros aspectos.

Pero si se definen algunas características y servicios más puntuales para estas redes de acceso, se podrá facilitar la toma de decisiones. Por ejemplo, si la cantidad de información a transportar es muy alta, si las distancias a cubrir no son grandes, si el medio usado es el aire, si el efecto de los fenómenos atmosféricos que inciden en la propagación no es muy determinante, si existen restricciones en normatividad y uso de frecuencias, y en últimas si el cliente considera que los costos de instalación y operación no son altos, entonces debería escogerse tecnologías que usen luz y se propaguen en el aire. 


\section{Problema de investigación}

Actualmente es posible implementar tecnologías que utilizan la luz visible para establecer la comunicación evitando algunos de los problemas que presentan las tecnologías cuyas portadoras están en la gama de las microondas y que se encuentran dentro de los sistemas de comunicaciones ópticas (OWC: Optical Wireless Communications). Desde el punto de vista de la gestión del espectro, la luz visible ofrece grandes anchos de banda que en la actualidad aún no han sido regulados en ninguna parte del mundo, por consiguiente, para ciertas aplicaciones pueden ser válidas porque además de ser inalámbricas (lo que permite movilidad) cuentan con buenas prestaciones en anchos de banda, velocidad de información y bajos costos (Bertolín, 2013).

Las tecnologías VLC (Visible Light Communications) y FSO (Free Space Optical) pertenecen a esta familia y son las que precisamente queremos analizar. Ambas se usan para la transmisión de datos de alta velocidad a frecuencias portadoras entre 400 y $800 \mathrm{THz}$ mediante el uso de dispositivos LED a través del espacio libre. VLC es unidireccional, mientras FSO es bidireccional.

La pregunta que mejor define el problema sería: ¿Un análisis comparativo de las tecnologías VLC y FSO ayuda a los ingenieros de telecomunicaciones en la toma de decisiones para asesorar a proveedores de servicios de acceso, teniendo en cuenta criterios técnicos y de economía?

\section{Objetivos de investigación \\ Objetivo general}

Realizar un análisis comparativo de las tecnologías VLC y FSO considerando aspectos técnicos y funcionales para generar un documento guía que ayude en la toma de decisiones a consultores y proveedores de servicios de telecomunicaciones en redes de acceso.

\section{Objetivos específicos}

- Describir las tecnologías VLC y FSO teniendo en cuenta criterios técnicos y funcionales en la transmisión de información.

- Analizar los parámetros que definen las redes de acceso con el fin de verificar la oportunidad de uso de las tecnologías VLC y FSO.

- Definir los aspectos técnicos, de instalación y calidad que se deben tener en cuenta en la prestación de servicios de telecomunicaciones en redes de acceso.

\section{Marco teórico}

\section{VLC}

La tecnología VLC (Visible Light Communications) utiliza la propagación de la luz visible del espectro de frecuencias con el fin de poder transmitir información de forma unidireccional y bidireccional a todo tipo de dispositivos tecnológicos como Smartphone, tabletas, PC portátiles y PC de mesa.

La limitación más importante del VLC es que actualmente sólo es posible disponer de gran ancho de banda a poca distancia de cobertura de la luz. VLC cuenta con la ventaja de que el ancho de banda no se comparte, por lo que si se ofrecen $100 \mathrm{Mbps}$, todos los usuarios de esa habitación dispondrán de esa capacidad. 
La óptica de espacio libre (FSO: Free Space Optical) como VLC es una tecnología de comunicación óptica que utiliza la propagación de la luz para transmitir información a través del espacio. FSO es una transmisión inalámbrica punto a punto con línea de vista que usa diodos emisores de luz (LED) o laser de alta potencia como fuente de transmisión de datos. Esta tecnología toma la señal de información digital y la modula con un rayo de luz infrarrojo para enviarla al receptor a través del espacio libre.

La tecnología FSO no requiere de licencia para utilizarse y es completamente inmune a interferencias radioeléctricas o electromagnéticas.

El propósito de este proyecto es estudiar ambas tecnologías con el fin de analizarlas detalladamente, comparando varios factores de cada una como la atenuación, interferencia, ancho de banda, velocidad, distancia, equipos, etc. Y a través de este estudio determinar cuál de las dos tecnologías (VLC y FSO) es más conveniente y eficaz para implementarla en un futuro.

\section{Redes de acceso}

Se conoce como red de acceso la parte de las redes que conecta los usuarios finales, ya sean residenciales o corporativos, a las redes de las operadoras de telecomunicaciones, aunque también está muy extendida la denominación última milla.

El término de última milla se comenzó a utilizar en telefonía para referirse a la conexión entre el abonado y la central telefónica. A esta conexión también se la conoce como bucle de abonado. Todas las conexiones entre los abonados y las centrales forman la llamada red de acceso. La red de última milla o red de acceso más extensa que existe en la actualidad sigue siendo el cableado telefónico (Gonzales, 2012).

\section{Metodología}

Este proyecto se realizará desarrollando una investigación de tipo cualitativo con características descriptivas de los parámetros físicos y de propagación, así como los aspectos técnicos y funcionales en su puesta en operación de las dos tecnologías de telecomunicaciones que se analizan. Para lograrlo se acudirá a la búsqueda de documentos en bases de datos creíbles que aborden el problema desde las perspectivas enunciadas en los objetivos.

La documentación de filtrará teniendo en cuenta criterios de pertenencia, actualidad y profundidad con el fin de extraer la información necesaria para definir y analizar la mejor forma de diseñar y entender una red de acceso mediante el uso de las tecnologías VLC o FSO.

\section{Desarrollo de la investigación}

\section{VLC}

Actualmente, entre las redes inalámbricas que cuentan con un gran ancho de banda, se puede considerar la tecnología 4G, utilizada para la telefonía móvil, que según la International Mobile Telecommunications-Advanced (IMT-Advanced), su ancho de banda se emplea entre los 100 Mbps cuando está en movimiento, y 1 Gpbs en reposo. En el 
presente, se cuenta con una nueva tecnología como VLC, la cual utiliza la propagación de la luz visible del espectro de frecuencias con el fin de poder transmitir información de forma unidireccional y bidireccional a todo tipo de dispositivos tecnológicos como Smartphone, tabletas, PC portátiles, PC de mesa, etc., con un gran ancho de banda (Riva, 2015).

La comunicación en VLC se origina en el transmisor debido a la variación en la intensidad de la luz, generando una diferenciación en la amplitud de voltaje de los pulsos de datos binarios que ingresan al modulador óptico, los cuales habitualmente son diodos LED de alta velocidad que se encuentran en componentes que ofrecen luz visible. Los datos son enviados a través de pulsos sucesivos de luz en modo apagado y encendido, reconociendo si es un uno binario ("1") o un cero binario ("0"), de acuerdo a la codificación empleada (Yanes, 2016).

\section{FSO}

Un sistema óptico de espacio libre (FSO), es un sistema inalámbrico laser que utiliza luz infrarroja y está diseñado para interconexiones de dos puntos situados en una línea de visión directa (LOS), que se propagan a través del espacio libre.

FSO utiliza la propagación de la luz visible o infrarroja para transmitir información a través de dos o más puntos. Esta tecnología utiliza como fuente de transmisión diodos emisores de luz o laser, los cuales no requieren un medio guiado, para los haces de luz, a través de cables ópticos. Durante el proceso de recepción, dichos haces operan alrededor de los Tera Hertz en el espectro, centrándose en un lente de recepción conectado a un receptor de alta sensibilidad a través de un cable de fibra óptica (CAIZALUISA, 2008).

\section{Principales hallazgos}

\begin{tabular}{|c|c|}
\hline VLC & FSO \\
\hline $\begin{array}{l}\text { - El espectro de la luz es } 10 \text { mil veces } \\
\text { más grande comparado con el es- } \\
\text { pectro actual de ondas de radio para } \\
\text { comunicaciones inalámbricas. }\end{array}$ & $\begin{array}{l}\text { Exento a interferencias de ra- } \\
\text { diofrecuencia y del espectro } \\
\text { electromagnético. }\end{array}$ \\
\hline $\begin{array}{l}\text { Los diodos LED son eficientes con } \\
\text { respecto al consumo de energía, } \\
\text { además son de bajo presupuesto y } \\
\text { logran transmitir datos a velocidades } \\
\text { de los Gigabits por segundo. }\end{array}$ & $\begin{array}{l}\text { - Logra alcanzar velocidades de } \\
\text { transferencia hasta los } 2.5 \text { Gi- } \\
\text { gabits por segundo. }\end{array}$ \\
\hline $\begin{array}{l}\text { Debido a que los haces de luz no so- } \\
\text { brepasan las paredes, la información } \\
\text { no puede ser interceptada, generan- } \\
\text { do así un mayor nivel de seguridad. } \\
\text { Adicionalmente, gracias a que esta } \\
\text { tecnología no interfiere con los sis- } \\
\text { temas electrónicos, puede ser usada } \\
\text { en aviones, hospitales y demás luga- } \\
\text { res sensibles a las interferencias. }\end{array}$ & $\begin{array}{l}\text { - La información puede trans- } \\
\text { mitirse cifradamente, lo cual } \\
\text { permite proporcionar un gra- } \\
\text { do de seguridad aún mayor. }\end{array}$ \\
\hline
\end{tabular}

Tabla 1. Características Principales de las tecnologías VLC y FSO Fuente: autoría propia.

(Tabla 1)

La tabla 1 muestra las características principales de cada una de las tecnologías, halladas durante el desarrollo del proyecto. 
Bibliografía

Bertolín, J. A. (1 de abril de 2013). Conectrónica tecnología y elementos de conexión y conectividad. Obtenido de https://www.conectronica.com/tecnologia/ seguridad/identificacion-y-analisis-de-la-tecnologia-vlcli-fi-desde-la-perspectiva-de-su-ciberseguridad-ciberprivacidad

CAIZALUISA, E. J. (julio de 2008). DISEÑO DE UNA RED CON TECNOLOGIA LASER FREE SPACE OPTICS (FSO), PARA EL CAMPUS DE LA ESCUELA POLITECNICA NACIONAL Y SU COMPARACION CON LA RED DE FIBRA OPTICA. QUITO.

Gonzales, M. (5 de noviembre de 2012). Redes Telemáticas. Obtenido de http://redestelematicas.com/ la-ultima-milla/

Riva, A. (2015). Transmisión inalámbrica de señales mediante el espectro de luz visible. Bolovia.

Yanes, J. (20 de junio de 2016). OpenMind. Obtenido de https://www.bbvaopenmind.com/li-fi-la-nueva-frontera-de-las-comunicaciones/?utm_source=materia\&utm_medium=web\&utm_content=pildora_redirect 